\title{
Jankov Formula and Ternary Deductive Term
}

\author{
Alex Citkin \\ Metropolian Telecommunications, New York, NY, USA \\ acitkin@gmail.com
}

Jankov (characteristic) formulas were introduced 50 years ago in [7] and proved to be a very useful tool for studying a broad range of logics, e.g. intermediate, modal, fuzzy, relevant, many-valued, etc. All these different logics have one thing in common: in one or the other form they admit the deduction theorem. From the standpoint of algebraic logic it means that their corresponding varieties have a ternary deductive (TD) term (4]). It is natural to try to extend the notion of characteristic formula to such varieties and, thus, to apply this notion to an even broader class of logics, namely, to the logics whose algebraic semantic is a variety with a TD term. We give such a generalization below.

Basic definitions. We consider the varieties of algebras in a finite signature $\Sigma$. A variety $\mathcal{V}$ is said to have a TD term, $t d(x, y, z)$, if for any algebra $\mathscr{A} \in \mathcal{V}$ and any a, b, c, d $\in \mathscr{A}$

$$
t d(\mathrm{a}, \mathrm{a}, \mathrm{b})=\mathrm{b} \text { and } t d(\mathrm{a}, \mathrm{b}, \mathrm{c})=t d(\mathrm{a}, \mathrm{b}, \mathrm{d}) \text { if }(\mathrm{c}, \mathrm{d}) \in \theta(\mathrm{a}, \mathrm{b}),
$$

where $\theta(\mathrm{a}, \mathrm{b})$ is a congruence generated by the elements $\mathrm{a}, \mathrm{b}$.

We will use the notation a (or $\underline{x}$ ) to denote a list of elements $\mathrm{a}_{1}, \ldots, \mathrm{a}_{n}$ (or a list of variables $\left.x_{1}, \ldots, x_{n}\right)$. If $\underline{x}, y$ are lists of variables of the same length by $t d(\underline{x}, y, z)$ we denote the iterated TD term $t d\left(x_{1}, y_{1}, t d\left(x_{2}, y_{2}, \ldots, t d\left(x_{n}, y_{n}, z\right) \ldots\right)\right)$. Then (1) can be generalized [4] as follows:

$$
t d(\underline{\mathrm{a}}, \underline{\mathrm{a}}, \mathrm{b})=\mathrm{b} \text { and } t d(\underline{\mathrm{a}}, \underline{\mathrm{b}}, \mathrm{c})=t d(\underline{\mathrm{a}}, \underline{\mathrm{b}}, \mathrm{d}) \text { if }(\mathrm{c}, \mathrm{d}) \in \theta(\underline{\mathrm{a}}, \underline{\mathrm{b}}) .
$$

The transition from (1) to 22 is a transition from principal to finitely generated congruences.

Recall that an algebra $\mathscr{A}$ is called subdirectly irreducible (s.i.) if the intersection $\mu(\mathscr{A})$ of all non-trivial congruences of $\mathscr{A}$ is a non-trivial congruence, and $\mu(\mathscr{A})$ is called a monolith. In every variety that has a TD term and, hence ([4), enjoys EDPC, every s.i. finitely presentable (f.p.) algebra $\mathscr{A} \in \mathcal{V}$ defines a splitting $\left(\mathcal{V}_{1}, \mathcal{V}_{2}\right)$, that is, $\mathcal{V}_{1}=\mathcal{V}(\mathscr{A})$ is a subvariety of $\mathcal{V}$ generated by $\mathscr{A}$ and $\mathcal{V}_{2}=\overline{\mathcal{V}}(\mathscr{A})$ is a co-splitting variety containing any such subvariety $\mathcal{V}^{\prime} \subset \mathcal{V}$ that $\mathscr{A} \notin \mathcal{V}^{\prime}$. Given the sets of identities $\mathcal{I}_{1}$ and $\mathcal{I}_{2}$ and a variety $\mathcal{V}$, we say that $\mathcal{I}_{1}$ and $\mathcal{I}_{2}$ are (semantically) $\mathcal{V}$-equivalent (in written $\mathcal{I}_{1} \simeq_{\mathcal{V}} \mathcal{I}_{2}$ ) if $\mathcal{I}_{1}$ and $\mathcal{I}_{2}$ define in $\mathcal{V}$ the same subvariety. If $\mathcal{I}_{i}=\left\{\mathrm{i}_{i}\right\}, i=1,2$ the identities $\mathrm{i}_{1}$ and $\mathrm{i}_{2}$ are called $\mathcal{V}$-equivalent and we write $\mathrm{i}_{1} \simeq \mathcal{V} \mathrm{i}_{2}$

Definition 1. Let $\mathcal{V}$ be a variety with a TD term $t d$ and the equalities $\mathrm{t}_{1}=\mathrm{t}_{1}^{\prime}, \ldots, \mathrm{t}_{n}=\mathrm{t}_{n}^{\prime}$ define in $\mathcal{V}$ a finitely presentable non-trivial subdirectly irreducible algebra $\mathscr{A}$. Let

$$
\chi_{\mathcal{V}}(\mathscr{A}):=t d\left(\underline{\mathrm{t}}, \underline{\mathrm{t}}^{\prime}, r\right) \approx t d\left(\underline{\mathrm{t}}, \underline{\mathrm{t}}^{\prime}, r^{\prime}\right)
$$

where terms $\mathrm{r}, \mathrm{r}^{\prime}$ express two distinct elements generating $\mu(\mathscr{A})$. Then we call the identity $\chi_{\mathcal{V}}(\mathscr{A})$ a $\mathcal{V}$-characteristic identity of algebra $\mathscr{A}$.

It had been observed in $[3$ that in the varieties with EDPC (and, hence, in the varieties with a TD term) every f.p. s.i. algebra defines splitting. It is also well known that every co-splitting variety can be defined (axiomatized) by a single meet-prime identity. Using a TD term of a given variety, the following theorem explicitly presents this identity. 
Theorem 1. Let $\mathcal{V}$ be a variety with a TD term and an s.i. algebra $\mathscr{A}$ be finitely presented in $\mathcal{V}$. Then $\chi_{\mathcal{V}}(\mathscr{A})$ defines the co-splitting variety $\overline{\mathcal{V}}(\mathscr{A})$.

In other words, given an f.p. s.i. algebra $\mathscr{A} \in \mathcal{V}$, for any algebra $\mathscr{B} \in \mathcal{V}$

$$
\mathscr{B}=\chi_{\nu}(\mathscr{A}) \text { if and only if } \mathscr{B} \in \mathbf{S H} \mathscr{B}
$$

and for any identity $\mathrm{i}$

$$
\mathscr{A} \not \models \mathrm{i} \text { if and only if } \mathrm{i} \vDash_{\mathcal{V}} \chi_{\mathcal{V}}(\mathscr{A}) \text {. }
$$

Note, that, in contrast to Jankov formula (which is completely defined by an finite s.i. algebra), the characteristic identity is relative to a variety: an algebra may be not finitely presentable in a variety, but may be finitely presentable in a subvariety. For instance, there are subvarieties of Heyting algebras containing infinite f.p. s.i. algebras and, hence, one can construct a characteristic identity that does not correspond to any Jankov formula. The definition of the characteristic identity also depends on a particular set of defining relations, but from the above properties of characteristic identity it immediately follows that two different characteristic identities of given algebra are $\mathcal{V}$-equivalent.

If $\mathcal{H}$ is a variety of Heyting algebras, then $\chi_{\mathcal{H}}(\mathscr{A}) \simeq_{\mathcal{H}} J(\mathscr{A}) \approx \mathbf{1}$ where $J(\mathscr{A})$ is a Jankov formula of algebra $\mathscr{A}$ (cf. [7).

Optimal Axiomatization by Characteristic Identities. We recall from 9 that the rank of an identity is a number of distinct variables occurring in it. The axiomatic rank of a variety $\mathcal{V}$ is the least natural number $r$ (that we denote by $r_{a}(\mathcal{V})$ ) such that $\mathcal{V}$ can be defined (axiomatized) by identities of the rank not exceeding $r$.

Let $\mathcal{V}$ be a variety and $\mathcal{V}^{\prime}$ be a subvariety of $\mathcal{V}$ axiomatized by a set of axioms $A x$. Then we will call the set Ax optimal if

(a) Ax is independent;

(b) Every identity in Ax is $\wedge$-prime;

(c) Axioms from $\mathrm{Ax}$ contain no more than $r_{a}(\mathcal{V})$ distinct variables.

Proposition 2. Let $\mathrm{Ax}_{1}$ and $\mathrm{Ax}_{2}$ be two independent axiomatizations of a given variety $\mathcal{V}$ consisting of $\wedge$-prime identities. Then there is a 1-1-correspondence $\phi$ between $\mathrm{Ax}_{1}$ and $\mathrm{Ax}_{2}$ such that $\mathrm{i} \simeq \mathcal{V} \phi(\mathrm{i})$.

The following theorem extends to locally finite varieties with a TD term the properties known for Heyting algebras (intermediate logics cf. [10, 12, 2]).

Theorem 3. Let $\mathcal{V}_{0}$ be a locally finite variety with a TD term. Then every subvariety $\mathcal{V} \subsetneq \mathcal{V}_{0}$ admits an optimal axiomatization constructed of characteristic identities.

Theorem 4. Let $\mathcal{V}$ be a finitely axiomatized hereditarily finitely approximated variety with a $T D$ term. Then there is an algorithm that given a list of axioms defining $\mathcal{V}$ and an identity $\mathrm{i}$ decides whether $\mathrm{i}$ is $\wedge$-prime.

Corollary 5. If $\mathcal{V}$ is a locally finite variety with a TD term then every identity is $\mathcal{V}$-equivalent to a finite set of characteristic identities. Moreover, if $\mathcal{V}$ is finitely axiomatized there is an algorithm that by a list of axioms defining $\mathcal{V}$ and an identity i returns a finite set of characteristic identities $\mathcal{V}$-equivalent to i. 
Varieties not Axiomatizable by Characteristic Identities. Not every variety with a TD term can be axiomatized by characteristic identities. Thus not every identity is equivalent to a set of characteristic identities. The simple examples can be given by using pre-true identities. An identity i is called pre-true in an algebra $\mathscr{A}$ (cf. [8]) if $\mathscr{A} \models \mathrm{i}$, but i is refutable in every proper subalgebra and proper homomorphic image of $\mathscr{A}$. Obviously, a characteristic identity of any finite algebra is pre-true in this algebra.

Theorem 6. Let $\mathrm{i}$ be an identity pre-true in an infinite algebra $\mathscr{A}$ from a varaiety $\mathcal{V}$ with a $T D$ term. Then $\mathrm{i}$ is not $\mathcal{V}$-equivalent to any set of characteristic identities.

The examples of formulas pre-true in infinite Heyting algebras can be found in [8, 13. Thus, there are finitely axiomatizable varieties of Heyting algebras (intermediate logics) that cannot be axiomatized by Jankov formulas. In fact, cf. [11, there is a continuum of varieties of Heyting algebras not axiomatizable by Jankov formulas.

We say that an identity $\mathrm{i}$ is uniquely pre-true in an algebra $\mathscr{A}$ if $\mathrm{i}$ is pre-true in $\mathscr{A}$ and it is not pre-true in any algebra not isomorphic to $\mathscr{A}$. For instance, if $\mathscr{A}$ is a finite s.i. algebra then $\chi_{\nu}(\mathscr{A})$ is uniquely pre-true in $\mathscr{A}$. On the other hand, in the varieties of Heyting or interior algebras if $\left\{\mathscr{A}_{i}, i \in I\right\}$ is a finite set of finite s.i. algebras such that $\mathscr{A}_{i} \notin \mathbf{S H} \mathscr{A}_{j}$ for all $i \neq j(i, j \in I)$, then the formula $\bigwedge_{i \in I} J\left(\mathscr{A}_{i}\right)$ is pre-true in every algebra $\mathscr{A}_{i}$.

Theorem 7. Suppose $\mathcal{V}$ is a hereditarily finitely approximated variety with a TD term and $\mathrm{i}$ is an identity. Then $\mathrm{i}$ is $(\mathcal{V}$-equivalent with) a characteristic identity of some $\mathcal{V}$-algebra if and only if $\mathrm{i}$ is uniquely pre-true in $\mathcal{V}$.

Locally Characteristic Identities in Varieties with a TD term. Using a TD term one can extend the notion of locally characteristic formula in sense of [6] ( partial characteristic formulas in sense of [11]). A partial subalgebra $\mathscr{A}^{\prime}$ of algebra $\mathscr{A}$ is a subset $\mathscr{A}^{\prime} \subseteq \mathscr{A}$ of elements of $\mathscr{A}$ with fundamental operations of $\mathscr{A}$ restricted to $\mathscr{A}^{\prime}$.

With each partial algebra $\mathscr{A}^{\prime}$ we can associate a positive diagram, that is a set

$$
\begin{aligned}
\delta^{+}\left(\mathscr{A}^{\prime}\right)=\left\{f\left(x_{\mathrm{a}_{1}}, \ldots, x_{\mathrm{a}_{n}}\right) \approx x_{f\left(\mathrm{a}_{1}, \ldots, \mathrm{a}_{n}\right)} \mid \mathrm{a}_{1}, \ldots, \mathrm{a}_{n} \in \mathrm{A}^{\prime}, f \in \Sigma\right. \\
\\
\text { and } \left.f\left(\mathrm{a}_{1}, \ldots, \mathrm{a}_{n}\right) \text { is defined }\right\} .
\end{aligned}
$$

Let $\mathcal{V}$ be a variety with a TD term $t d(x, y, z)$. We say that a partial algebra $\mathscr{A}$ belongs to $\mathcal{V}$ (in written $\mathscr{A} \in \mathcal{V}$ ) if there is a 1-1-homomorphism of $\mathscr{A}$ in some algebra from $\mathcal{V}$. If a partial algebra $\mathscr{A}$ can be embedded in an s.i. algebra, we say that $\mathscr{A}$ is subdirectly irreducible. With each finite non-degenerate partial algebra $\mathscr{A}$ and each pair of distinct elements b, c $\in \mathscr{A}$ we associate a locally characteristic identity $\chi\left(\mathscr{A}, x_{\mathrm{a}}, x_{\mathrm{b}}\right)$ in variables $x_{\mathrm{a}} ; \mathrm{a} \in \mathscr{A}$ as follows.

Let $\delta^{+}(\mathscr{A})=\left\{t_{i} \approx t_{i}^{\prime} ; 1 \leq i \leq m ;\right\}$ be a diagram, $\underline{t}:=t_{1}, \ldots, t_{m}$ and $\underline{t}^{\prime}:=t_{1}^{\prime}, \ldots, t_{m}^{\prime}$. Then

$$
\chi\left(\mathscr{A}, x_{\mathrm{a}}, x_{\mathrm{b}}\right):=t d\left(\underline{t}, \underline{t}^{\prime}, x_{\mathrm{b}}\right) \approx t d\left(\underline{t}, \underline{t}^{\prime}, x_{\mathrm{c}}\right) .
$$

In other words, we construct a characteristic identity in the same way we did using defining relations for finitely presented algebras, but now as defining relations we take the diagram identities.

Let $F P S(\mathscr{A})$ denote the set of all finite partial subalgebras of $\mathscr{A}$. Then with an algebra $\mathscr{A}$ we associate the set

$$
\mathcal{X}(\mathscr{A}):=\left\{\chi\left(\mathscr{A}^{\prime}, x_{\mathrm{a}}, x_{\mathrm{b}}\right) \mid \mathscr{A}^{\prime} \in F P S(\mathscr{A}), \mathrm{a}, \mathrm{b} \in \mathscr{A}^{\prime} \text { and } \mathrm{a} \neq \mathrm{b}\right\} .
$$

The main property of the characteristic set is as follows. 
Theorem 8. Let $\mathcal{V}^{\prime}$ be a variety with a TD term, $\mathcal{V} \subseteq \mathcal{V}^{\prime}$ be a subvariety and $\mathscr{A} \in \mathcal{V}^{\prime}$ be a full algebra. Then $\mathscr{A} \in \mathcal{V}$ if and only if each identity $\mathrm{i} \in \mathcal{X}(\mathscr{A})$ is refuted in $\mathcal{V}$.

The corollary below immediately follows from the above theorem and the fact that $\mathscr{A} \forall$ $\chi\left(\mathscr{A}^{\prime}, x_{\mathrm{a}}, x_{\mathrm{b}}\right)$ for any finite partial subalgebra $\mathscr{A}^{\prime}$ of any s.i. algebra $\mathscr{A}$.

Corollary 9. If $\mathcal{V}$ is a variety with a TD term then every subvariety of $\mathcal{V}$ can be axiomatized by locally characteristic identities.

Moreover, the following holds

Theorem 10. (comp. [5][Theorem 9.43]) Let $\mathcal{V}$ be a variety with a TD term and $\mathrm{i}$ be such an identity that $\mathcal{V} \vDash \mathrm{i}$. Then there is a finite set $\mathcal{I}$ of locally characteristic identities $\mathcal{V}$-equivalent to i.

In the variety of Heyting algebras a locally characteristic formula of a finite partial algebra with fully definied $\wedge$ and $\rightarrow$ is equivalent to the identity representing Zakharyaschev's canonical formula (for a definition, see e.g. [5]). Since every finite partial subalgebra of Heyting algebra can be extended to a finite partial subalgebra in which operations $\wedge, \rightarrow$ are fully defined, from the Theorem 8 it easily follows that any intermediate logic can be axiomatized by canonical formulas (cf. [11, 1]).

\section{References}

[1] Guram Bezhanishvili and Nick Bezhanishvili. An algebraic approach to canonical formulas: intuitionistic case. Rev. Symb. Log., 2(3):517-549, 2009.

[2] Nick Bezhanishvili. Lattices of intermediate and cylindric modal logics. PhD thesis, Institute for Logic, Language and Computation University of Amsterdam, 2006.

[3] W. J. Blok and D. Pigozzi. On the structure of varieties with equationally definable principal congruences. I. Algebra Universalis, 15(2):195-227, 1982.

[4] W. J. Blok and D. Pigozzi. On the structure of varieties with equationally definable principal congruences. III. Algebra Universalis, 32(4):545-608, 1994.

[5] Alexander Chagrov and Michael Zakharyaschev. Modal logic, volume 35 of Oxford Logic Guides. The Clarendon Press Oxford University Press, New York, 1997. Oxford Science Publications.

[6] Alex Citkin. Characteristic Formulas of Partial Heyting Algebras. Log. Univers., 7(2):167-193, 2013.

[7] V. A. Jankov. On the relation between deducibility in intuitionistic propositional calculus and finite implicative structures. Dokl. Akad. Nauk SSSR, 151:1293-1294, 1963. English translation in Sov. Math., Dokl. 4, 1203-1204 (1963).

[8] A. V. Kuznetsov and V. Ja. Gerčiu. The superintuitionistic logics and finitary approximability. Dokl. Akad. Nauk SSSR, 195:1029-1032, 1970. (in Russian).

[9] A.I. Mal'cev. Algebraic systems. Die Grundlehren der mathematischen Wissenschaften. Band 192. Berlin-Heidelberg-New York: Springer-Verlag; Berlin: Akademie-Verlag. XII,317 p., 1973.

[10] D. Skvortsov. Remark on a finite axiomatization of finite intermediate propositional logics. $J$. Appl. Non-Classical Logics, 9(2-3):381-386, 1999. Issue in memory of George Gargov.

[11] Eugeneusz Tomaszewski. On sufficiently rich sets of formulas. PhD thesis, Institute of Philosophy, JagellonianUniversity, Krakov, 2003.

[12] Eugeniusz Tomaszewski. An algorithm for finding finite axiomatizations of finite intermediate logics by means of Jankov formulas. Bull. Sect. Logic Univ. Lódź, 31(1):1-6, 2002.

[13] Andrzej Wroński. Intermediate logics and the disjunction property. Rep. Math. Logic, 1:39-51, 1973. 